\title{
One-Dimensional Time Dependent Analysis of the Detachment Front in a Divertor Plasma: Roles of the Cross-Field Transport ${ }^{*}$
}

\author{
Makoto NAKAMURA $^{\text {a) }}$, Satoshi TOGO, Masanori ITO and Yuichi OGAWA \\ Department of Advanced Energy, Graduate School of Frontier Sciences, The University of Tokyo, Kashiwanoha 5-1-5, \\ Kashiwa, Chiba 277-8561, Japan
}

(Received 7 December 2010 / Accepted 17 February 2011)

\begin{abstract}
Results of one-dimensional time dependent analysis of a detachment front in a PDD plasma are reported. Effects of the cross-field particle and energy trasport in a divertor region on a partially detached plasma are particularly discussed. It was found that such transport effects in the divertor region can prevent the detachment fronts from moving upstream, and that the position of the detachment front in a steady state is thermally unstable against the neutral density in the divertor region.
\end{abstract}

(C) 2011 The Japan Society of Plasma Science and Nuclear Fusion Research

Keywords: divertor detachment, detachment front, thermal stability, cross-field transport, one-dimensional model

DOI: $10.1585 /$ pfr.6.2403098

\section{Introduction}

Reduction of divertor heat load is a crucial issue for next generation fusion reactors such as ITER [1] and DEMO [2]. Plasma operation with detached divertors is considered to be a promising way to resolve this issue. For example, a partially detached divertor (PDD) regime will be one of the ITER operation senarios $[3,4]$ and has been used for the design of Slim-CS [5,6]. However, the plasma operation with divertor detachment involves a crucial issue that should be resolved; detachment fronts of plasma parameters, e.g. density, temperature and particle and heat fluxes, should be "captured" in a divertor region. Otherwise, cold neutrals and impurities would be transported upstream and cool a core plasma region. It is, therefore, crucial for divertor heat handling to elucidate plasma/impurity/neutral conditions on thermal instability of a detachment front and what physical processes can broaden or shrink an operation window of plasma parameters with divertor detachment that is thermally stable.

Divertor detachment is characterized by three types of plasma energy loss mechanisms: various kinds of impurity radiations, ionizations and radiative and three-body recombinations [7]. In these three types of energy loss mechanisms, the ionizations could not destabilize a position of a detachment front since $\mathrm{d}\langle\sigma v\rangle_{\text {ion }} / \mathrm{d} T>0$ in the plasma temperature range of interest: a few $\mathrm{eV}<T<$ several hundreds of $\mathrm{eV}$. (Here, $\langle\sigma v\rangle_{\text {ion }}$ is the ionization reaction rate and $T$ is the plasma temperature.) On the other hand, energy losses due to the impuritiy radiations and recombinations could destabilize a detachment front. In fact,

\footnotetext{
author'se-mail: nakamura.makoto@jaea.go.jp

*) This article is based on the presentation at the 20th International Toki Conference (ITC20).

a) Present address: Japan Atomic Energy Agency, 2-166 Oaza-ObuchiAza-Omotedate, Rokkasho, Kamikita, Aomori 039-3212, Japan
}

the derivatives of the impurity radiation energy loss rates due to carbon, neon and argon with respect to temperature, $\mathrm{d} L_{\mathrm{C}} / \mathrm{d} T, \mathrm{~d} L_{\mathrm{Ne}} / \mathrm{d} T$ and $\mathrm{d} L_{\mathrm{Ar}} / \mathrm{d} T$, are negative in the temperature ranges of $9 \mathrm{eV}<T<40 \mathrm{eV}, 40 \mathrm{eV}<T<200 \mathrm{eV}$ and $30 \mathrm{eV}<T<60 \mathrm{eV}$, respectively [8]. Here, $L_{\mathrm{C}}, L_{\mathrm{Ne}}$ and $L_{\mathrm{Ar}}$ are the plasma energy loss rates due to carbon, neon and argon impurities, respectively. The derivatives of reaction rates of the radiative and three-body recombinations with respect to temperature, $\mathrm{d}\langle\sigma v\rangle_{\mathrm{rr}} / \mathrm{d} T$ and $\mathrm{d}\langle\sigma v\rangle_{3 \mathrm{~b}} / \mathrm{d} T$, are negative in the temperature range of interest $[9,10]$. While thermal instability against the impurity radiations (i.e. MARFE) has been well studied [11-19] so far, conditions under which thermal instability due to the recombination energy losses occurs have not been studied enough for fusion reactor design. There are, nevertheless, some pioneering works. Krasheninnikov et al. have found that the detachment front is thermally destabilized when $Q_{\mathrm{H}}<Q_{\text {rec }}$ where $Q_{\mathrm{H}}$ is the total power input which can reach a recombination region and $Q_{\text {rec }}$ is the power sink due to the recombinations [7]. The similar power balance condition has been found independently by one-dimensional (1D) plasma fluid simulations by Nakazawa et al [20]. Goswami et al. have found that there is a correlation between the detachment front position and $Q_{\perp \mathrm{SOL}} / S_{\perp \mathrm{SOL}}$ (the ratio of cross-field power and particle sources from the core to the scrape-off layer (SOL)) [21]. However, in order to establish control methods for thermal stability of the detachment front, one need not only such a power balance condition but also an operational window of the plasma density and temperature and neutral particle conditions. Elucidation of physical mechanisms which can stabilize or destabilize the detachment front are also needed.

In this paper we show results of our one-dimensional time dependent analysis of a detachment front in a PDD 
plasma. Particularly, it is shown that cross-field heat and particle transport in a divertor region can prevent the detachment front from moving upstream. Such cross-field transport in a divertor region could occur in a PDD plasma.

\section{Model}

We performed time-dependent simulations of movement of detachment fronts in a PDD plasma by using the "multi-layer" (ML) 1D model [22]. Figure 1 is a schematic picture of this model. The basic idea is to put two flux tubes adjacent to each other. In order to reproduce a partially detached state observed in experiments (for example [23]), the inner flux tube, adjacent to the core plasma, should be detached from the divertor plate, while the outer one should be attached. In Fig. $1 \Delta_{\mathrm{AD}}$ and $\Delta_{\mathrm{DD}}$ are widths of the attached and detached tubes, respectively. A plasma fluid of each layer is described by the 1D transport equations (for example see [20,22]). Particle and heat transport across each layer are approximated as source or loss terms in the 1D transport equations. The source/loss terms in the particle, momentum and energy equations of each tube, $S$, $M$ and $Q$, are given as follows, respectively;

$$
\begin{aligned}
S= & S_{\perp \mathrm{SOL}}+S_{\perp \mathrm{div}} \\
& +n n_{\mathrm{n}}\langle\sigma v\rangle_{\mathrm{ion}}-n^{2}\left(\langle\sigma v\rangle_{\mathrm{rr}}+\langle\sigma v\rangle_{3 \mathrm{~b}}\right), \\
M= & -m n v\left\{n_{\mathrm{n}}\langle\sigma v\rangle_{\mathrm{cx}}+n\left(\langle\sigma v\rangle_{\mathrm{rr}}+\langle\sigma v\rangle_{3 \mathrm{~b}}\right)\right\}, \\
Q= & Q_{\perp \mathrm{SOL}}+Q_{\perp \mathrm{div}}-\left(\frac{1}{2} m v^{2}+\frac{3}{2} T\right) n n_{\mathrm{n}}\langle\sigma v\rangle_{\mathrm{cx}} \\
& -\left(\frac{1}{2} m v^{2}+3 T\right) n^{2}\left(\langle\sigma v\rangle_{\mathrm{rr}}+\langle\sigma v\rangle_{3 \mathrm{~b}}\right) \\
& -I_{\text {ion }} n n_{\mathrm{n}}\langle\sigma v\rangle_{\mathrm{ion}}-n^{2} \xi_{\mathrm{imp}} L(T) .
\end{aligned}
$$

Here, $n, v, T$ and $\langle\sigma v\rangle$ are the plasma density, velocity and temperature and the rate coefficient of an atomic process, respectively. In the transport equations, it is assumed that $n=n_{\mathrm{e}}=n_{\mathrm{i}}, v=v_{\mathrm{e}}=v_{\mathrm{i}}$ and $T=T_{\mathrm{e}}=T_{\mathrm{i}}$, where the subscripts e and i represent electrons and ions, respectively. The neutral density is represented by $n_{\mathrm{n}}$. Here, $S_{\perp \mathrm{SOL}}$ and $Q_{\perp \text { SOL }}$ are the cross-field particle and energy source terms, respectively, which might be outward, in the SOL region (from the core to the SOL), respectively, while $S_{\perp \text { div }}$ and $Q_{\perp \text { div }}$ are the cross-field particle and energy source terms in the divortor region. The constant $I_{\text {ion }}(=30 \mathrm{eV})$ is the electron loss energy due to excitation and ionization. The coronal equilibrium model is used for the impurity radiation loss, where $\xi_{\text {imp }}$ is the impurity fraction, and $L(T)$ is the impurity cooling rate dependent on impurity species. In this work carbon is the only impurity species and we set $\xi_{\text {imp }}=4.5 \%$ which is a typical value of many tokamak experiments (for example, see [24]).

The cross-field source terms in the SOL region, $S_{\perp \mathrm{SOL}}$ and $Q_{\perp \mathrm{SOL}}$, are important contributor to occurence of the plasma detachment [20, 21]. Hutchinson showed that $Q_{\perp \text { SOL }}$ can stabilize the detachment front of the parallel heat flux moving upstream [14]. In the "onion skin" model [25] the cross-field source terms are taken into ac-

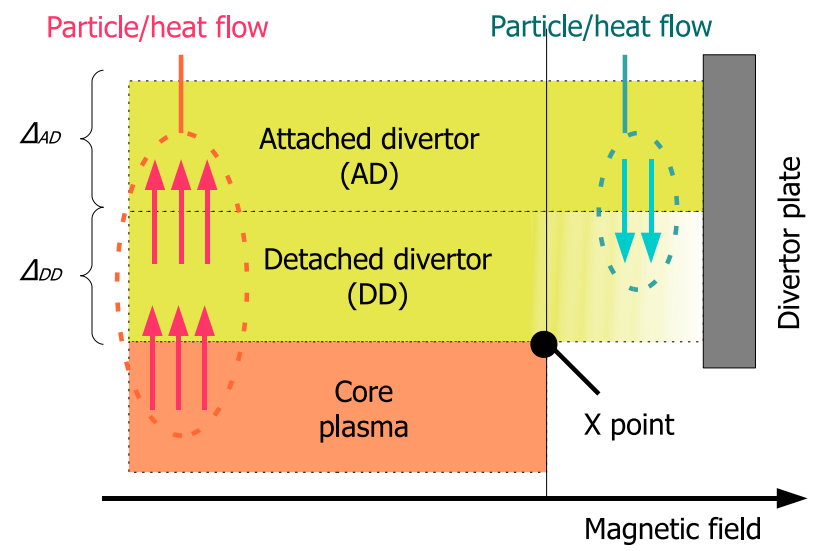

Fig. 1 The schematic picture of the multi-layer 1D model.

count only in the SOL region. On the other hand, effects of the cross-field source terms in the divertor region, $S_{\perp \text { div }}$ and $Q_{\perp \text { div }}$, on stability of the detachment front had not been studied so far. Recently, we showed that $Q_{\perp \text { div }}$ can decrease the speed of the detachment front upstream, but in our previous work $Q_{\perp \text { div }}$ is given an assumed value uniformly in the divertor region [22].

We extended our previous simulation study; we analyzed the attached and detached tubes simultaneously, where $S_{\perp \text { div }}$ and $Q_{\perp \text { div }}$ are modeled as follows;

$$
\begin{aligned}
& S_{\perp \mathrm{div}} \approx \frac{\Gamma_{\perp \mathrm{div}}}{\Delta_{\mathrm{div}}} \approx-\frac{D_{\perp \mathrm{div}}\left(n^{(\mathrm{det})}-n^{(\text {att })}\right.}{\Delta_{\mathrm{DD}} \Delta_{\Gamma}}, \\
& Q_{\perp \mathrm{div}} \approx \frac{q_{\perp \mathrm{div}}}{\Delta_{\mathrm{div}}} \approx-\frac{\chi_{\perp \mathrm{div}} \bar{n}\left(T^{(\mathrm{det})}-T^{(\mathrm{att})}\right)}{\Delta_{\mathrm{DD}} \Delta_{q}} .
\end{aligned}
$$

Here, the superscripts "det" and "att" represent the detached and attached tubes, respectively. The particle and heat diffusion coefficients are represented by $D_{\perp \text { div }}$ and $\chi_{\perp \text { div }}$, respectively. The characteristic lengths of the density and temperature gradients along the radial direction are $\Delta_{\Gamma}$ and $\Delta_{q}$, respectively. In Eq. (4) $\bar{n}$ is the average density along the cross-field direction. In the present analyses it is assumed that $\Delta_{\Gamma}=\Delta_{q}=\Delta_{\mathrm{SOL}} / 2$ where $\Delta_{\mathrm{SOL}}$ is the width of SOL.

For the neutral density $n_{\mathrm{n}}$, we use the simple $1 \mathrm{D}$ diffusion model described in [26];

$$
\begin{aligned}
& n_{\mathrm{n}}=n_{\mathrm{n}, \mathrm{d}} \exp \left(-\frac{\zeta}{\lambda_{\mathrm{n}, \mathrm{d}}}\right), \\
& \lambda_{\mathrm{n}, \mathrm{d}}=\frac{v_{\mathrm{n}}}{n \sqrt{\langle\sigma v\rangle_{\mathrm{cx}}\langle\sigma v\rangle_{\mathrm{ion}}} \sin \theta},
\end{aligned}
$$

where $n_{\mathrm{n}, \mathrm{d}}$ and $\lambda_{\mathrm{n}, \mathrm{d}}$ are the neutral density and its decay length at a reference point, $\zeta$ is the effective length measured from the reference point (given in [26]), and $\theta$ is the angle at which a magnetic field line intersects the target. The neutral velocity $v_{\mathrm{n}}$ is corresponding to $2 \epsilon_{\mathrm{FC}} / 3$ where $\epsilon_{\mathrm{FC}}(=3.5 \mathrm{eV})$ is the Franck-Condon energy. In numerical implementation the value of $n_{\mathrm{n}}$ at the discrete mesh point $j+1, n_{\mathrm{n}, j+1}$, is expressed in the form

$$
n_{\mathrm{n}, j+1}=n_{\mathrm{n}, j} \exp \left(-\frac{\zeta}{\lambda_{\mathrm{n}, j}}\right),
$$


where $n_{\mathrm{n}, j}$ and $\lambda_{\mathrm{n}, j}$ are the values of $n_{\mathrm{n}}$ and $\lambda_{\mathrm{n}}$ at the mesh point $j$, respectively. At the divertor target we adopt the following condition:

$$
n_{\mathrm{n}, \mathrm{div}} v_{\mathrm{n}}=\eta_{\text {trap }}(n v)_{\mathrm{div}} \sin \theta+n_{\mathrm{n}, \mathrm{div}, \text { aux }} v_{\mathrm{n}},
$$

where $\eta_{\text {trap }}$ is the recycling rate, $(n v)_{\text {div }}$ is the plasma particle flux at divertor target, and $n_{\mathrm{n} \text {,div,aux }}$ is the auxiliary injected neutrals, e.g. gas puffing, near the divertor plate. Input parameters relevant to the neutrals are $\eta_{\text {trap }}, \theta$ and $n_{\mathrm{n}, \mathrm{div}, \text { aux }}$.

\section{Numerical Results}

In our analysis, ITER-like plasma parameters were used. The power and particle inputs from the core to the SOL are $80 \mathrm{MW}$ and $1.5 \times 10^{23} \mathrm{~s}^{-1}$, respectively. The surface area $A_{\mathrm{SOL}}$ is $\sim 640 \mathrm{~m}^{2}$ and the SOL width $\Delta_{\mathrm{SOL}}$ is $\sim 4.7 \times 10^{-2} \mathrm{~m}$; therefore, the cross-field energy and particle source terms in the SOL region, $Q_{\perp \mathrm{SOL}}$ and $S_{\perp \mathrm{SOL}}$, are estimated to be $2.67 \mathrm{MWm}^{-3}$ and $5.0 \times 10^{21} \mathrm{~m}^{-3} \mathrm{~s}^{-1}$, respectively. The ratio of the detached tube width to the SOL width is $\Delta_{\mathrm{DD}} / \Delta_{\mathrm{SOL}}=1 / 3$. The distance from the stagnation point $(z=0)$ to the divertor target $(z=L)$ is $L=100 \mathrm{~m}$, and the X point is set at $z=80 \mathrm{~m}$. As for input parameters relevant to the neutral particles, $\eta_{\text {trap }}=0.8$ and $\theta=2^{\circ}$. The auxiliary neutral densities in the detached and attached tubes at the divertor plate is $n_{\mathrm{n} \text {,div,aux }}^{\text {(det) }}=$ $4.1 \times 10^{19} \mathrm{~m}^{-3}$ and $n_{\mathrm{n} \text {,div,aux }}^{\text {(att) }}=3.5 \times 10^{19} \mathrm{~m}^{-3}$, respectively.

\subsection{Effect of the cross-field energy transport}

As a first step, we examined effects of $Q_{\perp \text { div }}$ on behaviors of detachment fronts of several plasma parameters in a PDD plasma. Here we set $S_{\perp \text { div }}=0$ to elucidate the $Q_{\perp \text { div }}$ effects. Snapshots of variations of $n$ and $T$ in time in the attached and detached tubes are shown in Figs. 2 (a)(d), where $\chi_{\perp}=1.0 \mathrm{~m}^{2} \mathrm{~s}^{-1}$. For comparison the $n$ and $T$ variations in time in the detached tube in the case of $Q_{\perp \text { div }}=0$ are shown in Figs. 2 (e)-(f). Further, the position of the detachment front, $X_{\text {det }}$, in a steady state in the case of $Q_{\perp \text { div }}=0$ as a function of the auxiliary neutral density at the divertor plate, $n_{\mathrm{n} \text {,div, aux }}$, is shown in Fig. 3 . In the attached tube the spatial profiles of $n$ and $T$ are not so varied from the respective initial profiles (Figs. 2 (a) and (b)). In the case of $Q_{\perp \text { div }}=0$, the detachment fronts in $n$ and $T$ move upstream and then reach the $X$ point $(x=80 \mathrm{~m})$. On the other hand, as shown in Figs. 2 (c) and (d), the non-zero $Q_{\perp \text { div }}$, expressed in Eq. (5), prevents the detachment fronts from moving upstream. This results are consistent with our previous analysis in which we analyzed only the detached tube [22]. Thus, the cross-field heat transport in the divertor region has been found to affect the thermal stability of the detachment fronts.

\subsection{Effect of the cross-field particle trans- port}

As a next step, we examined the $S_{\perp \text { div }}$ effects on behaviors of the detachment front in a PDD plasma by intro-
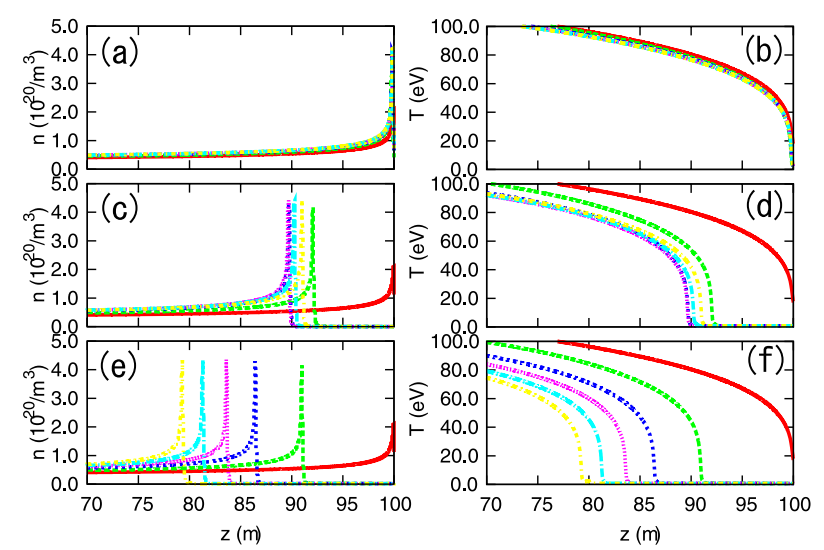

Fig. 2 Snapshots of the spatial profiles of $n$ and $T$ in the attached ((a) and (b)) and detached ((c) and (d)) tubes in the case of $S_{\perp \text { div }}=0$ and the non-zero $Q_{\perp \text { div }}$ expressed in Eq. (5). The $n$ and $T$ profiles in the case of $S_{\perp \text { div }}=Q_{\perp \text { div }}=0$ are also shown ((e) and (f)). The detachment fronts that move upstream in the detached tube are simulated. The time of each curve is at $t=0$ (red), 1.2 (green), 2.4 (blue), 3.7 (pink), 4.9 (turquoise) and 6.2 (yellow) s.

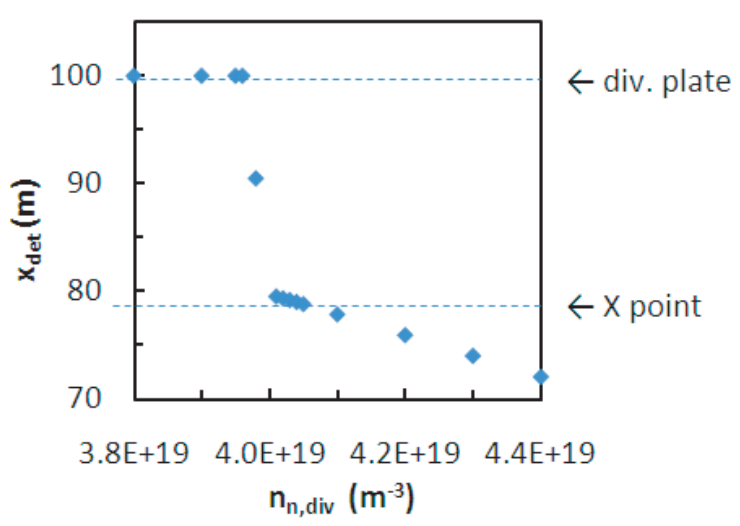

Fig. 3 The correlation between the auxiliary neutral density at the divertor plate, $n_{\mathrm{n}, \mathrm{div}, \text { aux }}$, and the position of the detachment front, $X_{\text {det }}$, in a steady state in the case of $Q_{\perp \text { div }}=0$.

ducing the non-zero $S_{\perp \text { div }}$, as well as the non-zero $Q_{\perp \text { div }}$, into the ML1D model. Simulation results are shown in Fig. 4. Figures 4 (a) and (b) are the case of $S_{\perp \text { div }}=0$ which are identical to Figs. 2 (c) and (d), respectively. Figures 4 (c)-(f) are the case of the non-zero $S_{\perp \text { div }}$ expressed in Eq. (4). In Figs. 4 (c) and (d) $D_{\perp}=0.5 \mathrm{~m}^{2} \mathrm{~s}^{-1}$; in Figs. 4 (e) and (f) $D_{\perp}=1.0 \mathrm{~m}^{2} \mathrm{~s}^{-1}$. The simulation results show that the cross-field particle transport, as well as the cross-field heat transport, prevent the detachment fronts from moving upstream, i.e. can affect the thermal stability of the detachment fronts.

This finding would be more surprising than the effect of the cross-field heat transport. This can be explained by the spacial profile of $S_{\perp \text { div }}$ in the detached tube that is shown in Fig.5. Figures 5 (a) and (b) correspond to Figs. 4 (c)-(d) and (e)-(f), respectively. As shown in Fig. 5, $S_{\perp \text { div }}<0$ around the detachment fronts, which means that there is an outward particle flux from the detached tube to 

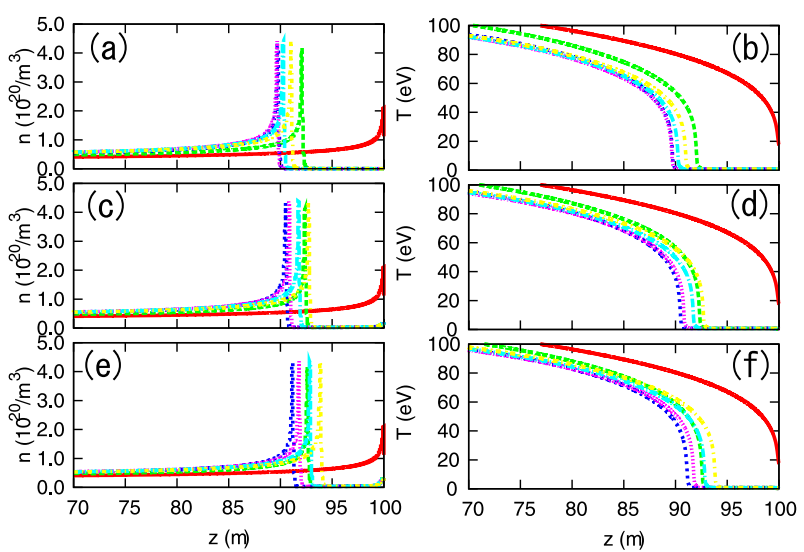

Fig. 4 Snapshots of the spatial profiles of $n$ and $T$ in the cases of the zero $S_{\perp \text { div }}\left((\mathrm{a})\right.$ and (b)) and the non-zero $S_{\perp \text { div }}((\mathrm{c})$-(f)) in the detached tube. The time step of each curve is the same as Fig. 2.

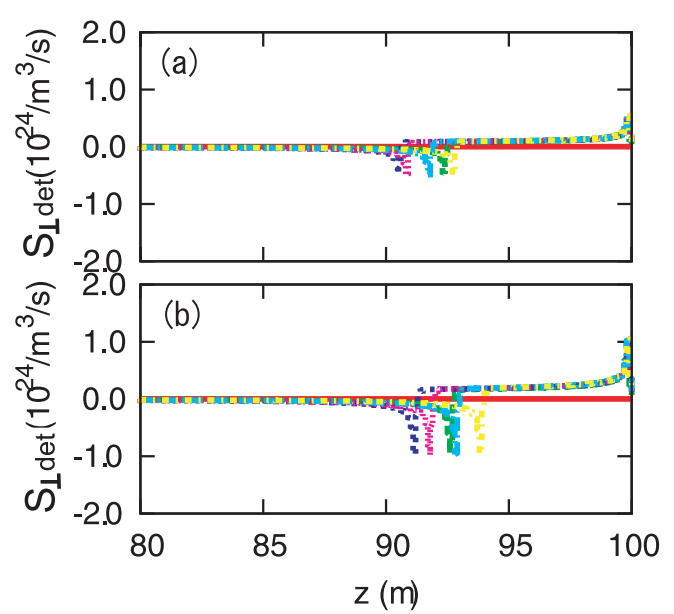

Fig. 5 Snapshots of the spacial profile of the cross field particle source term $S_{\perp \text { div }}$ in the divertor region of the detached tube with the cross-field particle diffusion coefficients of $D_{\perp}=0.5 \mathrm{~m}^{2} \mathrm{~s}^{-1}$ (a) and $D_{\perp}=1.0 \mathrm{~m}^{2} \mathrm{~s}^{-1}$ (b). The time step of each curve is the same as Figs. 2 and 4.

the attached one, opposite to the schematic picture shown in Fig. 1. The origin of such an outward particle flux is the peaking of the plasma density found in Fig. 4. Such a density peaking is formed by increase in the plasma density due to the ionizations and decrease in the plasma density due to the recombinations. Such an outward particle flux causes decrease in $n$ around the detachment front in the detached tube, leading to decrease in the plasma momentum and energy due to the recombination reactions because $M_{\text {rec }} \propto n^{2}$ and $Q_{\text {rec }} \propto n^{2}$. Here, $M_{\text {rec }}$ and $Q_{\text {rec }}$ are the plasma momentum and energy loss rates due to the recombinations, respectively. Hence, the movements of the detachment fronts toward upstream are hampered.

\section{Summary and Discussion}

We reported recent results of our one-dimensional time dependent analysis of the detachment fronts in a PDD plasma. We employed the ML1D model to describe a PDD plasma one-dimensionally. We found that the crossfield particle and heat transport in the divertor region can prevent the detachment fronts from moving upstream, i.e. such cross-field transport can hamper thermal instability of a PDD plasma. It is also found that the position of the detachment front in a steady state is thermally unstable against the neutral density at the divertor plate.

Finally, we make qualitative comparison of the simulation results reported here with experimental observations. In many tokamak experiments divertor detachment accompanies a high radiation peak which stagnates near the $\mathrm{X}$ point (for example [27]). On the other hand, as shown in Figs. 2 (e) and (f) and Fig. 4, the detachment front (accompanying the radiation peak) can move upstream and beyond the $\mathrm{X}$ point in the simulation cases of the zero cross-field transport. Such a situation contradicts the experimental observations. Our simulation results indicate that in interpreting such experimental observations of divertor detachment, it is important to model the cross-field transport in the divertor region.

[1] M. Shimada et al., Nucl. Fusion 47, S1 (2007).

[2] D.J. Ward, Plasma Phys. Control. Fusion 52, 124033 (2010).

[3] ITER Physics Expert Group on Divertor, Nucl. Fusion 39, 2391 (1999).

[4] L. Loarte et al., Nucl. Fusion 47, S203 (2007).

[5] K. Tobita et al., Nucl. Fusion 49, 075029 (2009).

[6] H. Kawashima et al., Nucl. Fusion 49, 065007 (2009).

[7] S.I. Krasheninnikov et al., J. Nucl. Mater. 266-269, 251 (1999).

[8] D.E. Post and R.V. Jensen, At. Data Nucl. Data Tables 20, 397 (1977).

[9] E. Hinnov and J.G. Hirshberg, Phys. Rev. 125, 795 (1962).

[10] Yu. Gordeev et al., Pisma Zh. Eksp. Theor. 25, 223 (1977).

[11] N. Ohyabu, Nucl. Fusion 9, 1491 (1979).

[12] B. Lipschultz et al., Nucl. Fusion 24, 977 (1984).

[13] J.A. Wesson and T.C. Hender, Nucl. Fusion 33, 1019 (1993).

[14] I.H. Hutchinson, Nucl. Fusion 34, 1337 (1994).

[15] Ph. Ghendrih, Phys. Plasmas 1, 1929 (1994).

[16] J. Kesner, Phys. Plasmas 2, 1982 (1995).

[17] S.I. Krasheninnikov et al., Phys. Plasmas 2, 2717 (1995).

[18] S.I. Krasheninnikov et al., Phys. Plasmas 4, 3741 (1997).

[19] S.I. Krasheninnikov et al., Phys. Plasmas 5, 2297 (1998).

[20] S. Nakazawa et al., Plasma Phys. Control. Fusion 42, 401 (2000).

[21] R. Goswami et al., Phys. Plasmas 8, 857 (2001).

[22] M. Nakamura et al., to be published in J. Nucl. Mater. (2011).

[23] G.F. Matthews, J. Nucl. Mater. 220-222, 104 (1995).

[24] P.C. Stangeby, The Plasma Boundary of Magnetic Fusion Devices (IOP Publishing, Bristol and Philadelphia, 2000) p.277.

[25] W. Fundamenski, J. Nucl. Mater. 290-293, 593 (2001).

[26] R. Schneider et al., Contrib. Plasma Phys. 46, 3 (2006).

[27] S. Konoshima et al., J. Nucl. Mater. 313-316, 888 (2003). 